\title{
Widening role of multidisciplinary treatment for rectal cancer: toward diversity of cancer care
}

\section{Yong Beom Cho}

Division of Colon \& Rectal Surgery, Department of Surgery, Samsung Medical Center, Sungkyunkwan University School of Medicine, Seoul, Korea

Received: December 22, 2021

Revised: December 24, 2021

Accepted: December 24, 2021

Corresponding author:

Yong Beom Cho

Department of Surgery, Samsung Medical Center, Sungkyunkwan University School of Medicine, 81 Irwon-ro, Gangnam-gu, Seoul 06351, Korea

Tel: +82-2-3410-1669

E-mail:

yongbeom.cho@samsung.com

This is an Open Access article distributed under the terms of the Creative Commons Attribution Non-Commercial License (https:// creativecommons.org/licenses/ by-nc/4.0/).
Advances in treatment modalities, including systemic therapy, local therapy, and technical development, have substantially improved survival rates in patients with colorectal cancer [1]. In addition, the rate of colorectal cancer diagnosis before 50 years of age has been increasing over the past two decades [2]. The number of cancer survivors who suffer from chronic morbidities that impair their quality of life has also increased and will continue growing. Therefore, precision medicine in the era of rectal cancer management aims to provide curative-intent treatment based on tumor characteristics and preserve the quality of life, especially bowel function with sphincter preservation or rectum sparing. Functional derangement was considerably significant after radical resection of rectal cancer. Abdominoperineal resection results in a colossal lifestyle change, and surgeons have attempted to preserve the sphincter. However, several patients experience bowel dysfunction even after sphincter preservation, which constitutes a series of symptoms. Although researchers have suggested various methods for evaluating and managing bowel dysfunction after rectal cancer surgery, treatment strategies need to be established.

Therefore, endoscopic removal or local excision has been widely accepted for early rectal cancer treatment, as oncologic outcomes are comparable to those after radical resection. Even in patients with advanced rectal cancer, neoadjuvant chemoradiotherapy resulted in complete tumor regression in a subset of patients, which led us to consider deferral surgery. Watch-andwait (WW) strategies have continuously gained approval in the Asia-Pacific region and Western countries [3]. Proper patient selection is critical for the organ-preserving strategies mentioned above.

In this issue of Precision and Future Medicine, three review articles have been published on rectal cancer management, in which interest has increased. Park and Baik [4] reviewed functional assessment methods and various treatments for bowel dysfunction. A series of symptoms associated with postoperative bowel dysfunction was typically called "low anterior resection syndrome (LARS)." Although different scoring systems have been proposed, each affords advantages and drawbacks. The LARS score questionnaire is a widely used tool. Young age, low anastomosis level, anastomotic leakage, and radiotherapy were related to the occurrence of LARS. Medical management, pelvic floor rehabilitation, and sacral nerve stimulation have been suggested for treating LARS. The authors also documented urinary and sexual dysfunctions, as reported in $20 \%$ to $40 \%$ of patients who undergo rectal cancer surgery. The authors concluded that surgeons should carefully perform sphincter-preserving rectal cancer surgery to ensure functional recovery of the remnant bowel and improve urologic and sexual 
PRECISION AND FUTURE MIEDICINE

Multidisciplinary treatment for rectal cancer

dysfunction.

In a review article by Son et al. [5], the authors reviewed the treatment of early rectal cancer, focusing on non-radical resection. Local excision, endoscopic resection, and neoadjuvant therapy have evolved to treat early rectal cancer and achieve organ preservation. Accurate preoperative diagnosis is critical for selecting an appropriate treatment strategy to obtain the best oncological outcome. Magnifying chromoendoscopy, narrowband imaging, and endoscopic ultrasonography would help discriminate deep submucosal invading lesions and successful endoscopic removal. Apart from the depth of invasion, lymph node metastasis is essential for prognosis, and pelvic magnetic resonance imaging can be used to evaluate lymph node metastasis, although its accuracy was shown to be heterogeneous. Pathological risk factors for resected specimens with endoscopic removal and local excision were crucial for determining further radical treatment. High-risk early rectal cancer is a potential candidate for radical resection. The lymph node metastasis rate was $<20 \%$. However, some patients need to consider surgery-related mortality, complications, and deterioration in the quality of life-related following radical resection. Adjuvant chemoradiotherapy may be considered in unsusceptible patients or those who refuse radical resection if a high risk of lymph node metastasis is identified after local excision or endoscopic removal. In a clinical trial setting, the goal of neoadjuvant treatment for rectal cancer is to preserve organs.

The final review article by Park [6] is on WW strategies for patients with rectal cancer who underwent radical resection. Neoadjuvant chemoradiotherapy with total neoadjuvant therapy resulted in clinical complete or near-complete regression of the primary tumor (good responder) in 15\% to $50 \%$ of patients. WW provides the benefits such as avoiding surgical complications and preserving the rectum. However, the current method for evaluating clinical response has limited accuracy, and the evaluation time remains unclear. Although numerous studies have reported comparable oncologic outcomes between WW and radical resection in good responders, evidence regarding oncologic outcomes remains insufficient. Several trials are underway to increase the number of patients with an excellent clinical response using increased radiation dose, the addition of chemotherapy, prolonged waiting time, and intensified chemotherapy. WW will play a crucial role in managing rectal cancer in good responders; therefore, we need to be flexible when accepting WW in clinical practice. Meanwhile, we need to remain cautious of WW, as it may deprive some patients of curative-in- tent treatment.

From these three review articles, readers will be able to learn more regarding current treatment trends for rectal cancer, aimed at obtaining good oncologic outcomes and maintaining the quality of life at the same time. It is also vital to understand the limitations of less extensive treatment and apply multimodal therapy in rectal cancer management to improve the quality of life of patients who undergo standard radical resection. Paradigm shifts are ongoing in the treatment of rectal cancer.

\section{CONFLICTS OF INTEREST}

No potential conflict of interest relevant to this article was reported.

\section{ORCID}

Yong Beom Cho https://orcid.org/0000-0002-9944-4706

\section{REFERENCES}

1. van Gijn W, Marijnen CA, Nagtegaal ID, Kranenbarg EM, Putter H, Wiggers T, et al. Preoperative radiotherapy combined with total mesorectal excision for resectable rectal cancer: 12-year follow-up of the multicentre, randomised controlled TME trial. Lancet Oncol 2011;12:575-82.

2. National Cancer Institute. SEER ${ }^{\star}$ Stat Database: November 2018 Submissions: Rate Sessions-Incidence-SEER 9 Regs Research Data with Delay Adjustment, Malignant Only, Nov 2018 Sub (1975-2016), Katrina/Rita Population Adjustment [Internet]. Bethesda (MD): SEER; 2018 [cited 2021 Dec 20]. Available from: https://seer.cancer.gov/datasoftware/documentation/seerstat/nov2018.

3. Huh JW, Maeda K, Liu Z, Wang X, Roslani AC, Lee WY. Current status of "watch-and-wait" rectal cancer treatment in Asia-Pacific Countries. Ann Coloproctol 2020;36:70-7.

4. Park EJ, Baik SH. Functional outcomes after sphincterpreserving surgeries for low-lying rectal cancer. Precis Future Med 2021;5:164-74.

5. Son KM, Lee IY, Cho SH, Park BS, Kim HS, Park SB, et al. Multidisciplinary treatment strategy for early rectal cancer. Precis Future Med 2021 Dec 27 [Epub]. https://doi.org/ 10.23838/pfm.2021.00163

6. Park IJ. Watch and wait strategies for rectal cancer. Precis Future Med 2021 Dec 27 [Epub]. https://doi.org/10.23838/ pfm.2021.00177 